\title{
Implantation of engineered conduction tissue in the rat heart
}

\author{
WENBO ZHANG ${ }^{1,2^{*}}$, XIAOTONG $\mathrm{LI}^{2,3^{*}}$, SHANQUAN SUN $^{4}$ and XI ZHANG ${ }^{2}$ \\ ${ }^{1}$ Class B (4) of Grade 2014, Department of Clinical Medicine, Chongqing Medical University, Chongqing 400016; \\ ${ }^{2}$ Institute of Biomedical Engineering, Second Military Medical University, Shanghai 200433; \\ ${ }^{3}$ Department of Anatomy, Shanghai Jiao Tong University School of Medicine, Shanghai 200011; \\ ${ }^{4}$ Department of Anatomy, Chongqing Medical University, Chongqing 400016, P.R. China
}

Received April 22, 2018; Accepted January 3, 2019

DOI: $10.3892 / \mathrm{mmr} .2019 .9933$

\begin{abstract}
Engineered conduction tissues (ECTs) are cardiac conduction tissues fabricated in vitro to allow for more precisely targeted in vivo transplantation therapy. The transplantation of ECTs may be ideal for the treatment of atrioventricular conduction block and could have a significant impact on the future application of biological pacemakers. However, there is little published information regarding the conduction function of ECTs in vivo. In the present study, ECTs were constructed by seeding cardiac progenitor cells (CPCs) into a collagen sponge and were then transplanted into animal hearts to determine whether they could act as an atrioventricular conduction pathway. The results demonstrated that the transplanted ECTs were adequately vascularized at the early stage of transplantation and could survive in the atrioventricular junction area of rats. A large number of myocardial tissue ( $\geq 29 \%$ of the new muscle fiber tissue formation area in the implanted ECTs) were observed by Masson's trichrome staining at 60 days post-transplantation. Positive staining for connexin-40, connexin-43, HCN2 and cTnT was exhibited during the period of 20 to 90 days post-transplantation. This result suggested that the transplanted ECTs formed gap junctions with the allogeneic myocardium and developed into cardiac conduction tissues with certain myocardial components. Electrocardiography (ECG) confirmed that there was a clear pre-excitation syndrome in the rats transplanted with ECTs during the period of 20 to 90 days post-transplantation.
\end{abstract}

Correspondence to: Professor Shanquan Sun, Department of Anatomy, Chongqing Medical University, 1 Yixueyuan Road, Chongqing 400016, P.R. China

E-mail: sunsq2151@sohu.com

Dr Xi Zhang, Institute of Biomedical Engineering, Second Military Medical University, 800 Xiangyin Road, Shanghai 200433, P.R. China E-mail: zhangxicyl1126@163.com

*Contributed equally

Key words: cardiac progenitor cells, atrioventricular conduction block, engineered conduction tissues, transplantation, biological pacemakers
The recovery rate in the rats implanted with ECTs was $61.54 \%$ within $1 \mathrm{~h}$ following atrioventricular block, and the heart rhythm following recovery was close to normal. By contrast, the recovery rate was only $4.17 \%$ in the rats implanted with blank collagen sponges (BCSs), and none of the sham rats exhibited atrioventricular block recovery. In conclusion, ECTs can survive and mechanically integrate with the allogeneic myocardium following transplantation into rat hearts. An atrioventricular accessory pathway similar to Kent bundles could be established between the atria and ventricles of rats following implantation. It is suggested that ECTs may be a potential substitution therapy for atrioventricular conduction block.

\section{Introduction}

Complete atrioventricular (AV) conduction block, a significant complication of numerous manifestations of heart disease, is a serious threat to human health. Biological pacemakers are of particular interest in cardiac conduction studies. Recent studies $(1,2)$ have focused on the use of cell transplants and gene transfer for AV conduction block therapy. However, neither gene transfer nor cellular transplantation have been able to reproduce the normal function of cardiac conduction. These methods only provide palliation of complete heart block. In particular, transgene expression is transient, and the cells do not remain at the desired injection site. Engineered conduction tissues (ECTs) are biological conduction tissues fabricated in vitro. Compared with the aforementioned methods, ECTs allow for more precisely targeted and reliable regenerative therapy. Therefore, the transplantation of ECTs may be useful for the treatment of AV conduction block. However, there is very little published information regarding the effectiveness of ECTs in vivo.

In 2006, Choi et al (3) used a tissue engineering approach to fabricate biocompatible, three-dimensional, collagen-based constructs that contained fetal rat myoblasts. These experiments provided proof of the principle that engineered tissue constructs are able to function as an electrical conduit and may offer a substitute treatment for conventional pacing therapy. Subsequently, Hou et al (4) demonstrated that anastomosis of the right auricle and right ventricle assisted by MSCs may be a future treatment for patients with complete AV block. Although the aforementioned studies created engineered 
tissue constructs as electrical conduits for AV conduction block therapy, the tissue constructs did not develop into cardiac conduction tissue or produce a physiological effect in vivo. In the two studies, the tissue constructs were used only as a method to deliver cells abundantly and conveniently to the heart. Therefore, little is currently known regarding the role that an engineered conduction tissue may serve in vivo.

ECTs may be fabricated by seeding the appropriate cells into scaffolds in vitro. Previously, we reported that $\mathrm{Nkx} 2.5^{+}$ cardiac progenitor cells (CPCs) derived from embryonic heart tubes were able to differentiate into cardiac cells, including cardiomyocytes, pacemaker cells and endothelial cells (5-7). In the present study, ECTs were created by seeding CPCs into a collagen sponge in order to investigate the feasibility that engineered conduction tissue could restore the normal rhythm of the heart in rats with atrioventricular block.

\section{Materials and methods}

Engineered conduction tissues were fabricated using rat CPCs. All the experimental procedures were conducted in accordance with the institutional guidelines for the care and use of laboratory animals of the Second Military Medical University (Shanghai, China) and conformed to the National Institutes of Health Guide for the Care and Use of Laboratory Animals. The methods used for culturing $\mathrm{Nkx} 2.5^{+} \mathrm{CPCs}$ were as previously described $(5,6)$. In brief, at embryonic day 11 heart tubes were dissected from a total of 27 female SD rats (14 weeks old, 270-320 g). The rats were obtained from the laboratory animal center of Second Military Medical University in Shanghai and housed in a room at $18-26^{\circ} \mathrm{C}$, with $40-70 \%$ relative humidity, and a $12-\mathrm{h}$ light/dark cycle. The animals had free access to food and water. The heart tubes were disaggregated in trypsin $0.25 \%$ EDTA (Gibco; Thermo Fisher Scientific, Inc., Waltham, MA, USA). The cells were pelleted by centrifugation at $400 \mathrm{x} \mathrm{g}$ for $5 \mathrm{~min}$ at room temperature, resuspended in culture medium with a $10^{5} / \mathrm{ml}$ cell density and seeded in 12-well cell culture plates (Corning Incorporated, Corning, NY, USA). The medium used was Dulbecco's modified Eagle's medium (HyClone; GE Healthcare Life Sciences, Logan, UT, USA), supplemented with $15 \%$ fetal bovine serum (Gibco; Thermo Fisher Scientific, Inc.), $20 \mu \mathrm{g} / 1 \mathrm{EGF}$ (R\&D Systems, Inc., Minneapolis, MN, USA), $10^{6}$ U/1 LIF (Chemicon International, Inc.; EMD Millipore, Billerica, MA, USA), $0.375 \% \mathrm{NaHCO}_{3}, 1 \%$ penicillin-streptomycin (Gibco; Thermo Fisher Scientific, Inc.) and $2 \mathrm{mM}$ L-glutamine (Amresco, LLC, Solon, OH, USA). After 5 or 7 days of primary culture, the cells were isolated using $0.25 \%$ trypsin for further experiments. The Nkx $2.5^{+} \mathrm{CPCs}$ were identified and selected as described previously $(5,6)$.

The CPCs were labeled with $10 \mu \mathrm{g} / \mathrm{ml}$ CM-Dil (Invitrogen; Thermo Fisher Scientific, Inc.) for $5 \mathrm{~min}$ at $37^{\circ} \mathrm{C}$ and $15 \mathrm{~min}$ at $4^{\circ} \mathrm{C}$. The ECTs were fabricated by seeding the CPCs in a collagen sponge (Wuxi Biot Bio-technology, Co., Ltd., Wuxi, China). A CPC suspension with a density of $10^{7} / \mathrm{ml}$ was added to the bottom of the 6 -well culture plate. The collagen sponge was placed in the cell suspension. After the cell suspension was absorbed by the sponge, the sponge was inverted so that the internal porosity of the sponge was completely filled with the cell suspension. The constructs were transferred into an incubator maintained at $37^{\circ} \mathrm{C}$ in $95 \%$ humidity and $5 \% \mathrm{CO}_{2}$ for $3 \mathrm{~h}$. Subsequently, the base medium with $10 \mu \mathrm{g} / \mathrm{l} \mathrm{bFGF}$ (R\&D Systems, Inc.) was added to the 6-well culture plate to immerse the constructs; the medium was changed every 3 days, and the cells were cultured for 2 weeks. The base medium contained DMEM supplemented with $15 \%$ fetal bovine serum (Gibco; Thermo Fisher Scientific, Inc.), 0.375\% $\mathrm{NaHCO}_{3}, 1 \%$ penicillin-streptomycin (Gibco; Thermo Fisher Scientific, Inc.) and $2 \mathrm{mM}$ L-glutamine (Amresco, LLC). The control groups were blank collagen sponges (BCSs) not seeded with CPCs. After 14 days, the ECTs and BCSs were harvested and used for subsequent implantation.

In vivo implantation in the rat atrioventricular junction area. A total of 70 male SD rats (18 weeks old) weighing between 350 and $400 \mathrm{~g}$ were prepared for surgery. The rats were obtained from the laboratory animal center of Second Military Medical University in Shanghai and housed in a room at $18-26^{\circ} \mathrm{C}$, with $40-70 \%$ relative humidity, and a 12 -h light/dark cycle. The animals had free access to food and water. The animals were divided into three groups: The ECTs $(n=26)$, BCSs $(n=24)$ and Sham $(n=20)$. Their anterior right-sided chests were opened in layers at the fifth intercostal space. Following incision of the pericardium above the right atrium, the epicardium of the atrium and the ventricle near the aorto-atrioventricular triangle was carefully removed (3). The prepared ECTs were implanted into the atrioventricular junction area by surgical thoracotomy. The implantation site in the myocardium below the epicardial surface was marked with 7-0 polypropylene suture (Yangzhou Fuda Medical Devices, Co., Ltd., Yangzhou, China). The chests were closed, and the animals were left to recover from anesthesia of $300 \mathrm{mg} / \mathrm{kg}$ chloral hydrate (Capot Chemical Co., Ltd., Hangzhou, China) and then returned to their cages. Throughout the surgery, the animals were ventilated with a respirator. A limb lead (left hind limb, right and left forelimb) surface electrocardiogram (ECG) monitored the cardiac rhythm using an MPA 2000 multiple biological signal analysis system (Shanghai Alcott Biotech, Co., Ltd., Shanghai, China).

Histological staining. The tissues surrounding the implantation site were excised and fixed in $4 \%$ paraformaldehyde for $36 \mathrm{~h}$ at room temperature. Next, the tissues were embedded in paraffin and sectioned at 4 or $6 \mu \mathrm{m}$. Following incubation at $60^{\circ} \mathrm{C}$ for $1 \mathrm{~h}$, the sections were immersed in xylene for $10 \mathrm{~min}$ and then in fresh xylene for a further $10 \mathrm{~min}$. The sections were treated to remove wax. The sections were then successively rehydrated in 100,95 or $80 \%$ ethanol and purified water for $3 \mathrm{~min}$ each for hematoxylin and eosin, Masson's trichrome and immunohistochemical staining. For the hematoxylin and eosin staining, the sections were stained with hematoxylin for $10 \mathrm{~min}$, differentiated with ethanol hydrochloride and transferred to eosin solution for $2 \mathrm{~min}$, all at room temperature. The vessel (diameter, $>30 \mu \mathrm{m}$ ) density was calculated as the number of vessels $/ \mathrm{mm}^{2}$. The density measure was determined from 6 randomly selected microscopic fields by a blinded observer (8). Masson's trichrome staining (Abcam, Cambridge, MA, USA) was performed according to the manufacturer's protocols. For immunohistochemical staining, the sections were incubated in fresh $3 \%$ hydrogen peroxide at room temperature for $10 \mathrm{~min}$ 

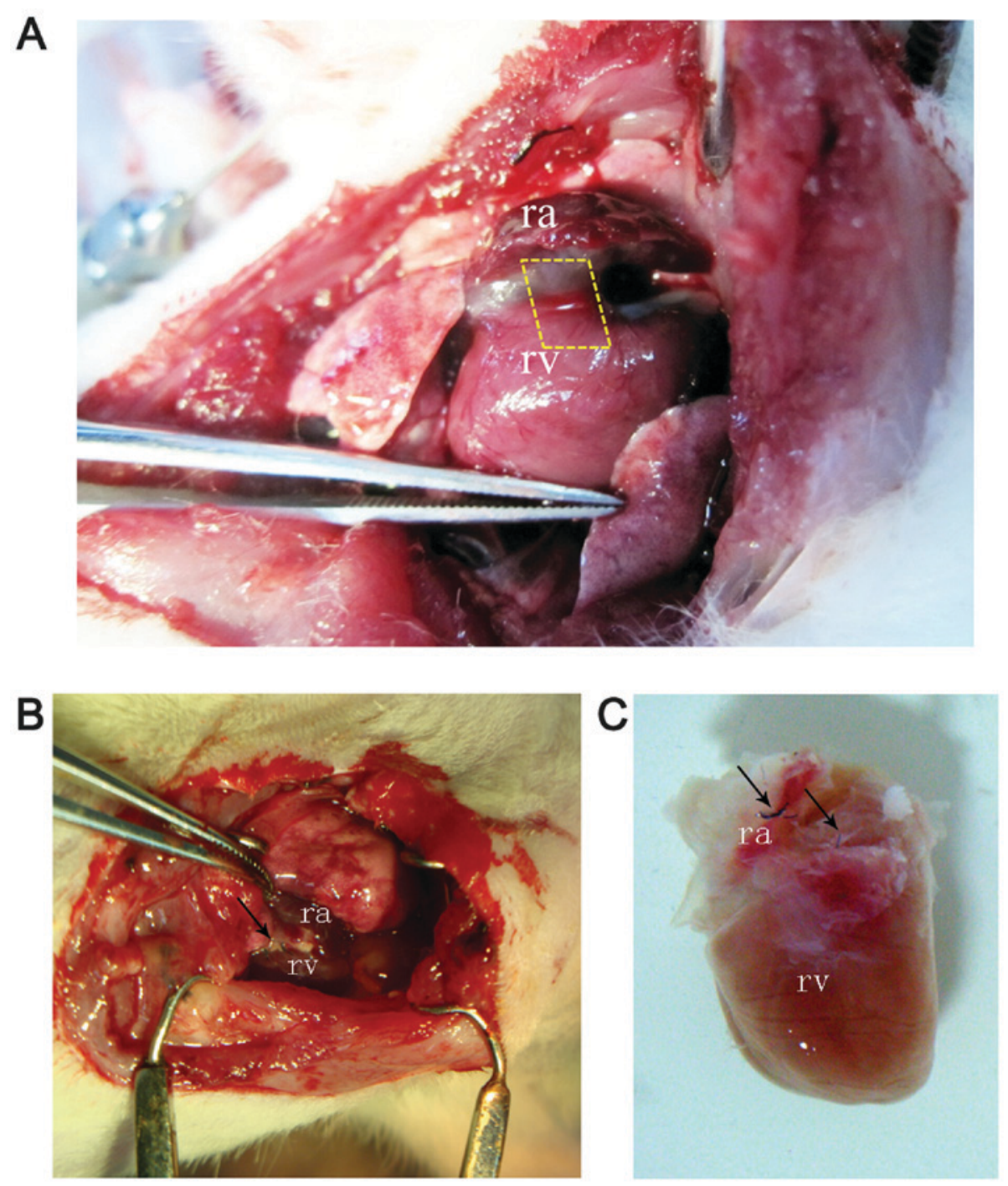

Figure 1. The atrioventricular groove was exposed by a right-sided thoracotomy at the fifth intercostal space. (A) The implantation site was located within the epicardial layer adjacent to the aorto-atrioventricular triangle; (B) the implanted ECTs in vivo were observed after 60 days; (C) and the implanted ECTs ex vivo were observed after 60 days. rv, right ventricle; ra, right atrium; ECTs, engineered conduction tissues.

to remove endogenous peroxidase blocking buffer. Then the sections were sufficiently eluted with PBS and goat serum (Thermo Fisher Scientific, Inc.) was added dropwise onto the slices at room temperature for $30 \mathrm{~min}$. Excess solution was discarded and the sections were incubated at $4^{\circ} \mathrm{C}$ overnight with primary antibodies, including rabbit anti-rat CD-31 (cat. no. BA2966; 1:200), Factor-VIII (cat. no. PB0273; 1:100) and VEGFR2 (cat. no. A00901; 1:500; Wuhan Boster Biological Technology, Ltd., Wuhan, China), followed by treatment with goat anti-rabbit peroxidase-conjugated secondary antibody (cat. no. ab6721; 1:1,000; Abcam) for $1 \mathrm{~h}$ at room temperature for visualization with 3-diaminobenzidine tetrahydrochloride. Additionally, the nuclei of the cultured cells were stained with hematoxylin for $10 \mathrm{~min}$ at room temperature. Subsequently, the sections were dehydrated and mounted with resinene. Finally, microscopic analysis was performed using an Olympus BH-2 light microscope (Olympus Corporation, Tokyo, Japan).

Immunofluorescence staining. To detect cardiac marker proteins of the implantation tissues, immunofluorescence staining was performed. The implantation tissues were excised, fixed with 4\% PFA for $36 \mathrm{~h}$ at room temperature and dehydrated in $30 \%$ sucrose in phosphate-buffered saline. Subsequently, the tissues were embedded in Tissue-Tek O.C.T compound (Sakura Finetech USA, Inc., Torrance, CA, USA) and sectioned at 5 micrometers at $-26^{\circ} \mathrm{C}$. The frozen sections were placed on poly-L-lysine-coated glass slides and stored in a $-20^{\circ} \mathrm{C}$ freezer. For the immunofluorescence staining, the sections were sufficiently eluted with PBS and goat serum (Thermo Fisher Scientific, Inc.) was added dropwise onto the sections at room temperature for $30 \mathrm{~min}$. Excess solution was discarded and the tissues were incubated with rabbit anti-connexin-40 (cat. no. 36-4900; 1:200; Invitrogen; Thermo Fisher Scientific, Inc.), connexin-43 (cat. no. C6219; 1:150; Sigma-Aldrich; Merck KGaA, Darmstadt, Germany), connexin-45 (cat. no. PA5-79311; 1:200; Invitrogen; Thermo Fisher Scientific, Inc.), HCN2 (cat. no. ab19346; 1:200; Abcam), Hcn4 (cat. no. AB5808; 1:200; Chemcon International, Inc.; EMD Millipore) and cTnT (cat. no. ab45932; 1:200; Abcam) at $4^{\circ} \mathrm{C}$ overnight. The control staining was performed by omitting the primary antibody. Following the sections being incubated with the FITC-conjugated anti-rabbit IgG 

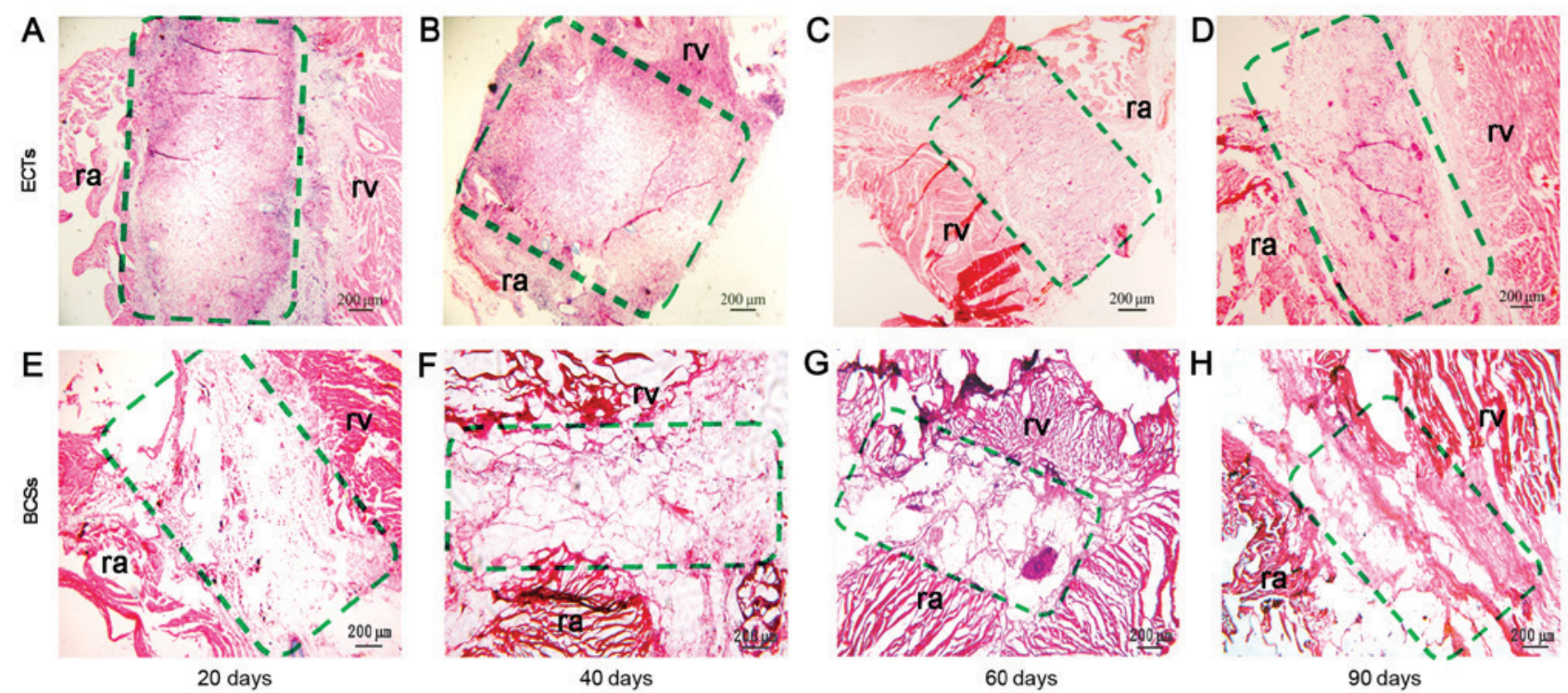

Figure 2. Gross observation of the implantation site by H\&E staining. Representative staining in the rats transplanted with ECTs at days (A) 20, (B) 40, (C) 60 and (D) 90 after implantation; and representative staining in the rats transplanted with BCSs at days (E) 20, (F) 40 , (G) 60 and (H) 90 following implantation. The green dotted line boxes show the implantation sites. rv, right ventricle; ra, right atrium; ECTs, engineered conduction tissues; BCSs, blank collagen sponges.

secondary antibodies (cat. no. F0382; 1:200; Sigma-Aldrich; Merck $\mathrm{KGaA}$ ) for $60 \mathrm{~min}$ at room temperature, fluorescence imaging was visualized using an Olympus IX70 fluorescence microscope (Olympus Corporation).

Atrioventricular block (AVB). A complete AVB was created at days 20,60 and 90 after the implantation of ECTs and BCSs by ethanol, as previously described by Lee et al (9). In brief, the rats were anesthetized by $300 \mathrm{mg} / \mathrm{kg}$ chloral hydrate (Capot Chemical Co., Ltd.) and $15 \mu 170 \%$ ethanol was injected into the myocardium $3 \mathrm{~mm}$ below the epicardial surface. The injection point was close to the epicardial fat pad between the aortic root and the right atrial wall of the rats. The reagents were injected twice in $10 \mathrm{~min}$. Six-lead surface ECGs (Alcott Biotech Co., Ltd., Shanghai, China) were used to monitor the cardiac rhythm.

Statistical analysis. Statistical analysis was conducted with SPSS version 21.0 (IBM Corp., Armonk, NY, USA). The average vessel (diameter $>30 \mu \mathrm{m}$ ) densities, presented as the mean \pm standard error of the mean, were compared using Dunnett's test. The recovery rate was compared using the $\chi^{2}$ test, followed by Fisher's exact test. $\mathrm{P}<0.05$ was considered to indicate a statistically significant difference.

\section{Results}

Implanted ECTs are capable of surviving and vascularizing in the heart. The heart was exposed by right-sided thoracotomy at the fifth intercostal space. Samples of 5x2x2 mm ECTs or BCSs were implanted into the AV groove. The implanted site was positioned within the epicardial layer adjacent to the aorto-atrioventricular triangle (Fig. 1A). Following a period of growth in vivo, the implanted ECTs were adequately combined with the host's myocardium. The implanted ECTs were associated with the atrium and the ventricle (Fig. 1B and C). The tissues surrounding the implantation site were excised and sectioned at 4 or 6 micrometers. Next, the sections were stained with $H \& E$. The results revealed that a large number of cells were gathered at the junction between the implants and the heart at days 20 and 40 after transplantation (Fig. 2A and B), but there were few cell clusters observed at the junction at days 60 and 90 after transplantation (Fig. 2C and D). It was suggested that these cells may be inflammatory cells. The number of cells (red staining) increased significantly, and the collagen sponges (slight staining) were gradually degraded in the implanted ECTs between 20 and 90 days post-transplantation (Fig. 3). The scaffold at the recipient site was completely degraded at 90 days post-transplantation. The transplanted tissues formed a cell-matrix structure similar to the host tissue at days 60 and 90 after transplantation. By contrast, there were fewer cells in the BCSs that failed to form a cell-matrix structure similar to the host tissue (Figs. 2 and 3). The vessel densities of the transplanted tissues were calculated, and the results revealed that the vessel number in the ECTs was greater than that of the BCSs at the implantation site at days 20 and 40 (Fig. 3). This observation was consistent with the result of Masson's trichrome staining, which revealed that ECTs formed more vessels than BCSs (Figs. 4 and 5). The ECT tissue was stained positive for CD-31, factor-VIII and VEGFR2 markers, indicating that there were vessels in the tissue (Fig. 6). It was suggested that the transplanted ECTs were adequately vascularized at the early stage of transplantation and could survive in the atrioventricular junction area of the rats. In addition, a large number of muscle fiber tissues were observed by Masson's trichrome staining of the transplanted ECTs at 60 and 90 days post-transplantation.

The transplanted ECTs develop into a phenotype similar to cardiac conduction tissues. The expression of connexin-40, connexin-43, connexin-45, Hcn2, Hcn4 and cTnT in the implanted tissue was detected by immunofluorescence 

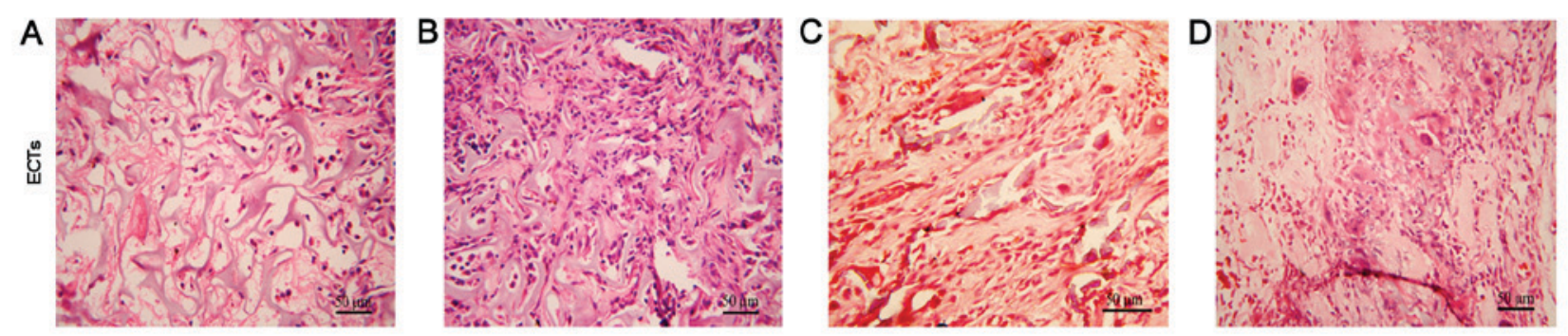

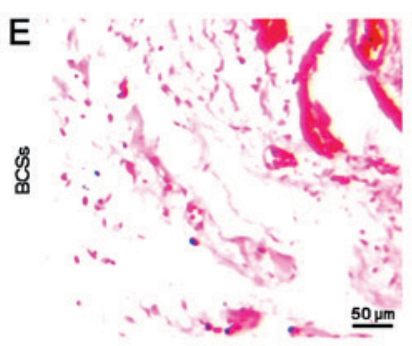

20 days

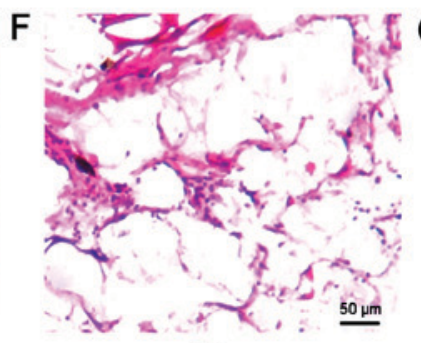

40 days

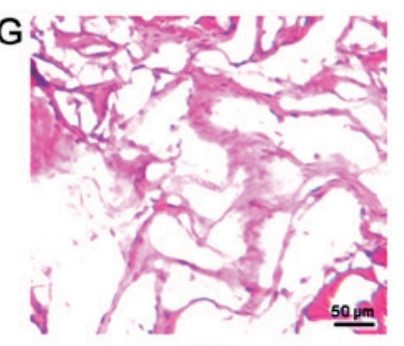

60 days

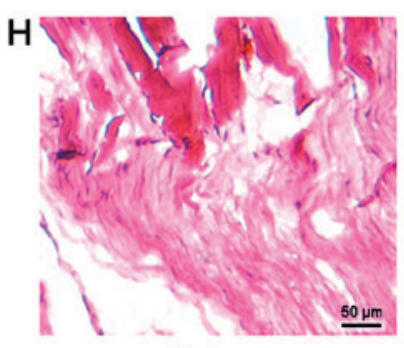

90 days

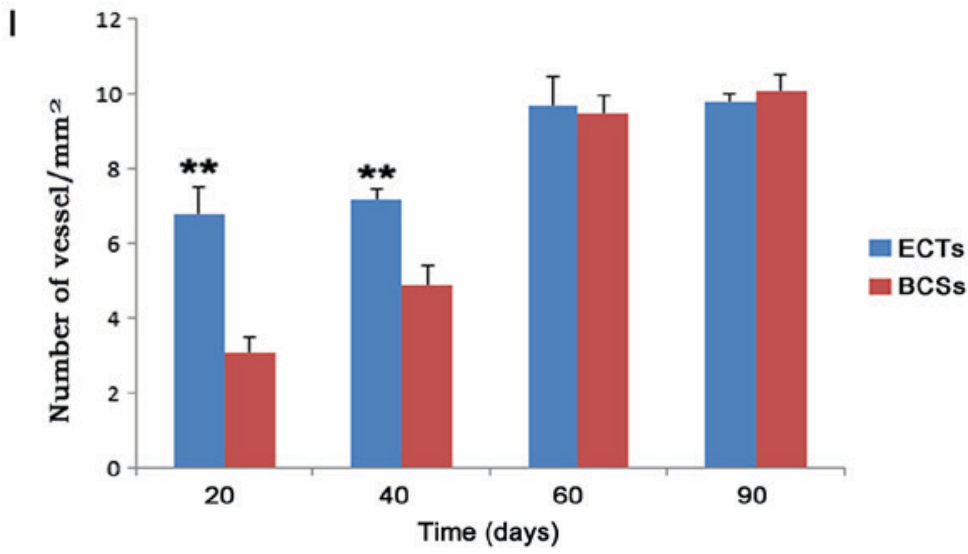

Figure 3. Detailed observation of the implantation site by H\&E staining. Representative staining in the rats transplanted with ECTs at days (A) 20, (B) 40, (C) 60 and (D) 90 following implantation; representative staining in the rats transplanted with BCSs at days (E) 20, (F) 40 , (G) 60 and (H) 90 following implantation; and (I) quantitative assessment for the vessel densities determined in 6 randomly selected microscopic fields by a blinded pathologist ( $\mathrm{n}=12$ ). rv, right ventricle; ra, right atrium; ECTs, engineered conduction tissues; BCSs, blank collagen sponges. ${ }^{* *} \mathrm{P}<0.01$, compared with corresponding values in the BCS group.
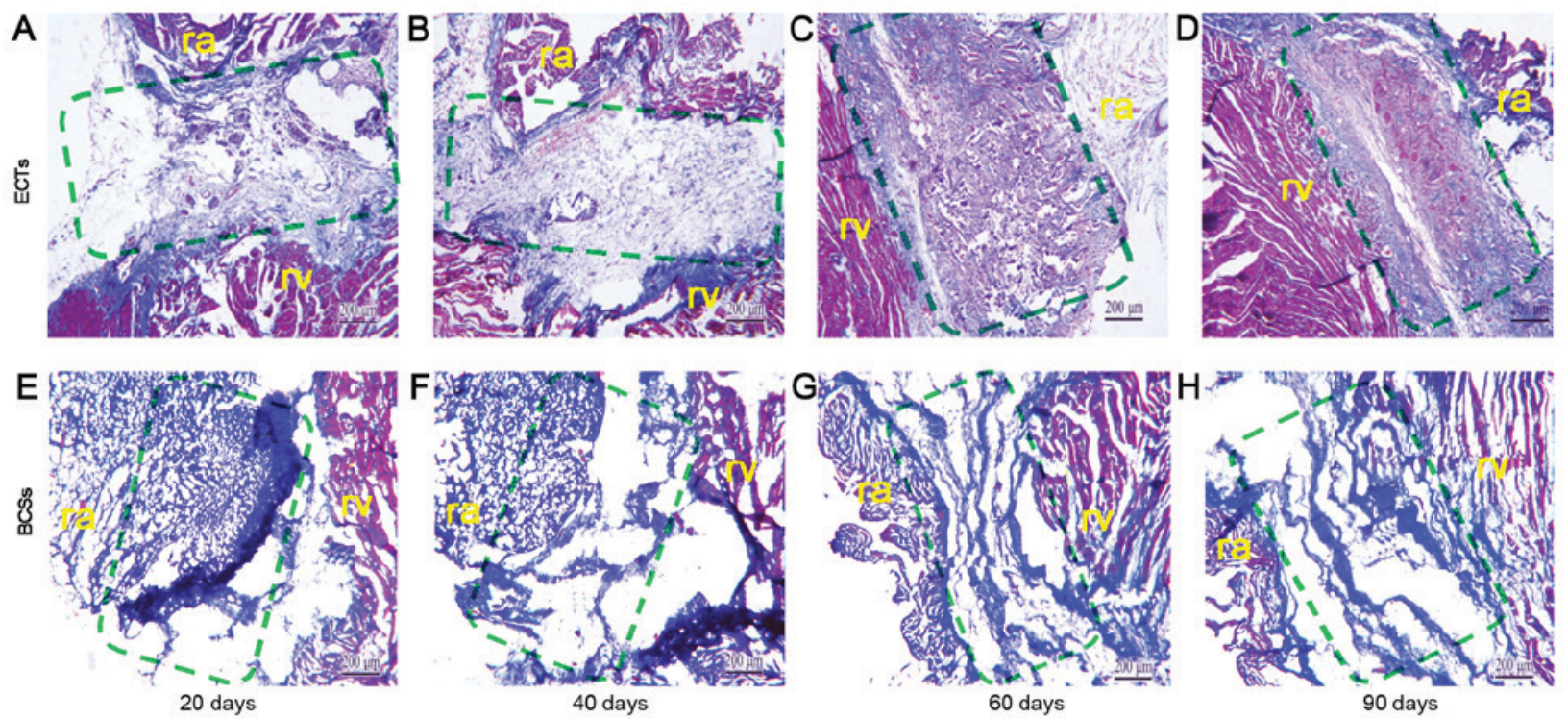

Figure 4. Gross observation of the implantation site by Masson's trichrome staining. Representative staining in the rats (n=26) transplanted with ECTs at days (A) 20, (B) 40, (C) 60 and (D) 90 after implantation; and representative staining in the rats (n=24) transplanted with BCSs at days (E) 20, (F) 40, (G) 60 and (H) 90 after implantation. The green dotted line boxes show the implantation sites; rv, right ventricle; ra, right atrium; ECTs, engineered conduction tissues; BCSs, blank collagen sponges. 

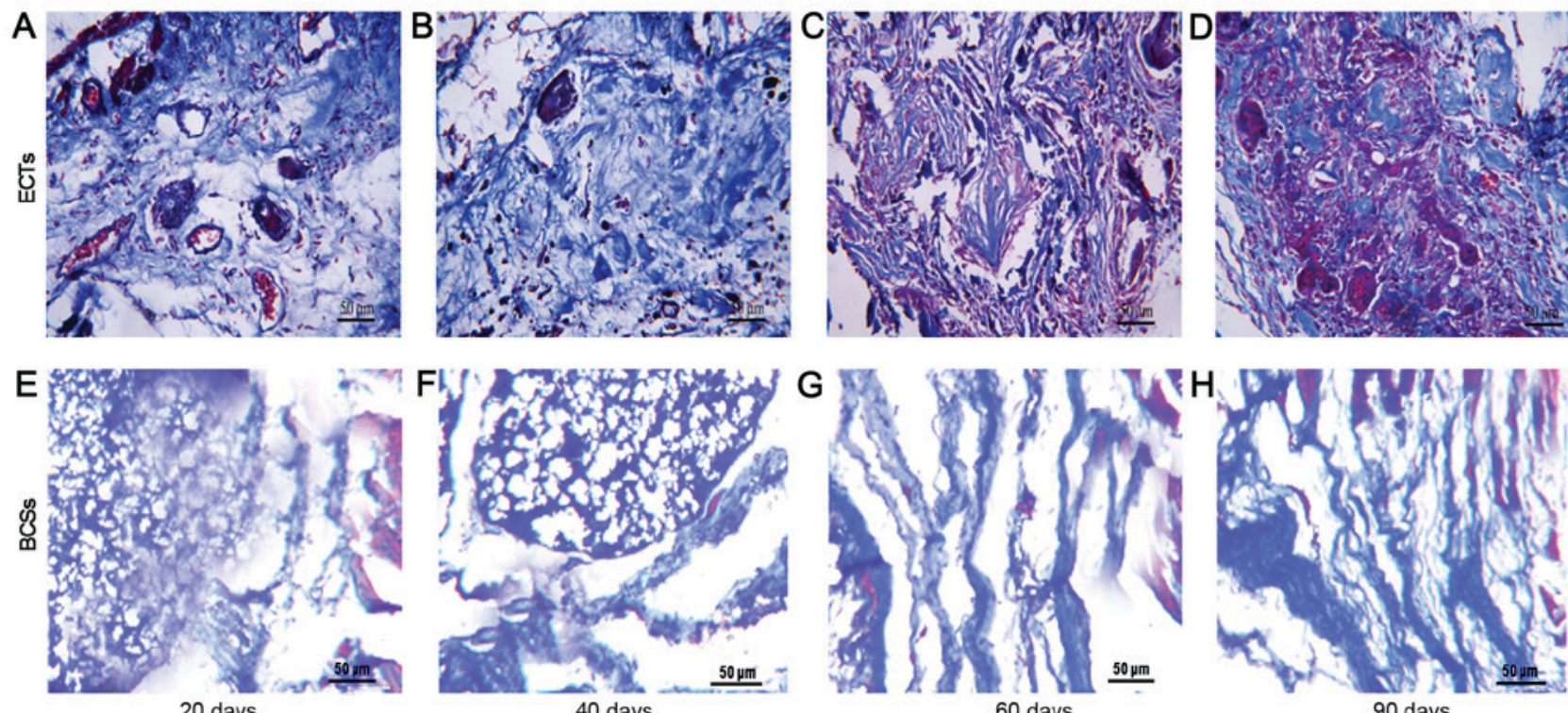

Figure 5. Detailed observation of the implantation site by Masson's trichrome staining. Representative staining in the rats (n=26) transplanted with ECTs at days (A) 20, (B) 40, (C) 60 and (D) 90 after implantation; and representative staining in the rats transplanted with BCSs (n=24) at days (E) 20, (F) 40, (G) 60 and $(\mathrm{H}) 90$ after implantation. rv, right ventricle; ra, right atrium; ECTs, engineered conduction tissues; BCSs, blank collagen sponges.
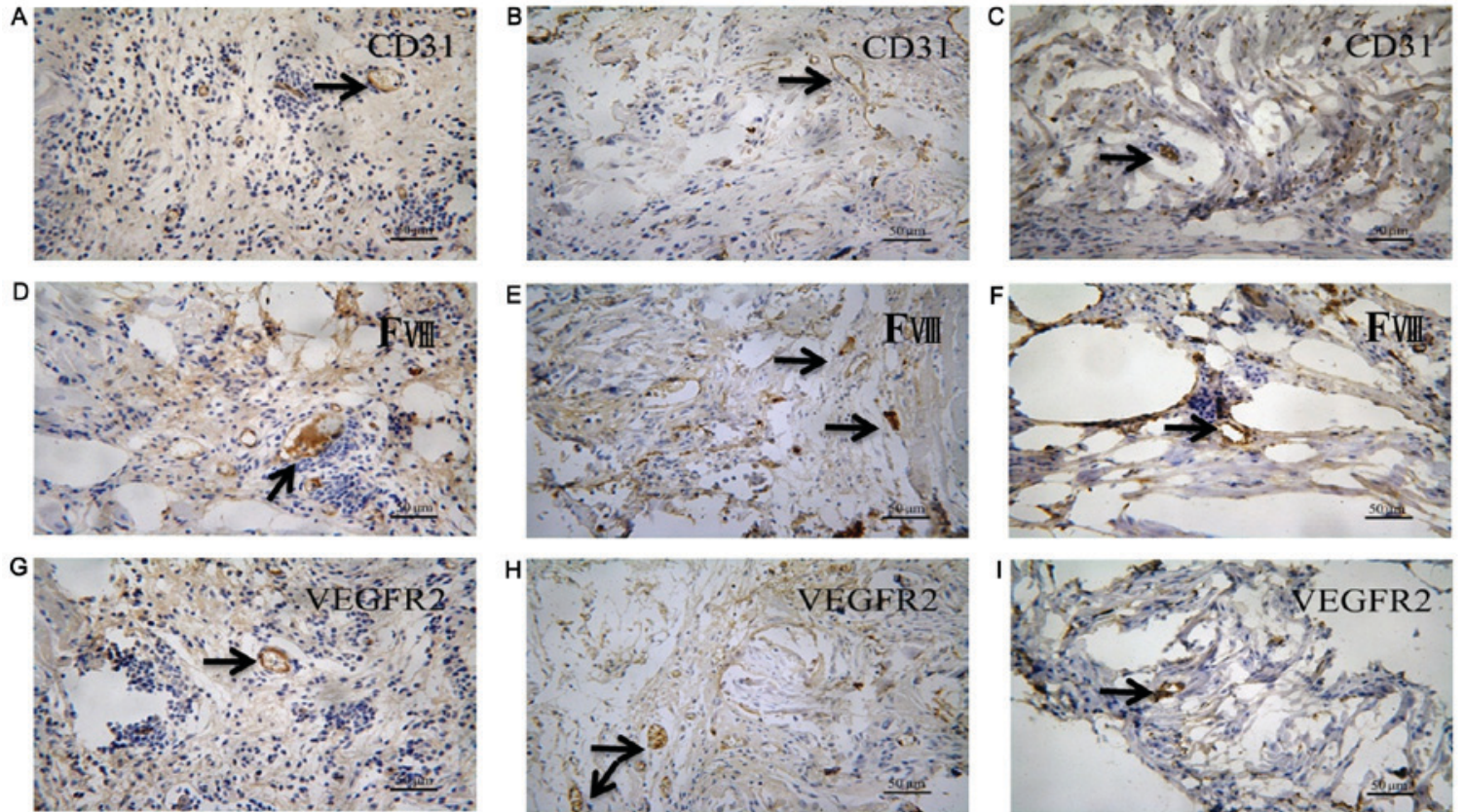

20 days

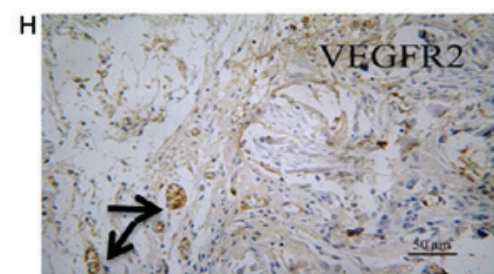

40 days

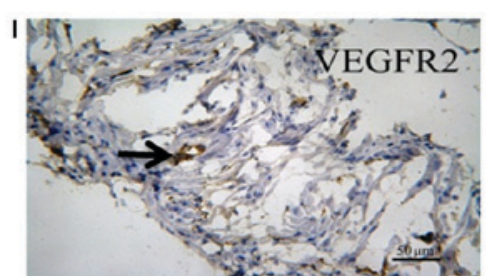

60 days

Figure 6. Immunohistochemical staining for the expression of vascular markers. Positive staining for CD-31 is shown at days (A) 20, (B) 40 and (C) 60 ; positive staining for Factor-VIII is shown at days (D) 20, (E) 40 and (F) 60; and positive staining of VEGFR2 is shown at days (G) 20, (H) 40 and (I) 60. The black arrows indicate the presence of the vascular markers (yellow). CD-31, cluster of differentiation 31; VEGFR2, vascular endothelial growth factor receptor 2.

staining. The results revealed that the transplanted ECTs exhibited stronger positive staining for connexin-40, connexin- 43 and Hen 2 during the period of 20 to 90 days posttransplantation. By contrast, the transplanted BCSs exhibited negative staining for the three proteins (Fig. 7A, B and C). The transplanted ECTs exhibited little positive staining for the molecular markers of pacemaker cells, including connexin-45 and Hcn4 (Fig. 7D and E). In addition, cTnT, as a marker of working myocardium, was positive in the transplanted ECTs and negative in the transplanted BCSs during the period of 20 to 90 days post-transplantation (Fig. 7F).

Transplanted ECTs provide atrioventricular conduction of the accessory pathway. ECG recordings were used to detect the atrioventricular conduction of the accessory pathway. The results revealed that there was an obvious pre-excitation syndrome in the rats transplanted with ECTs during the 
A
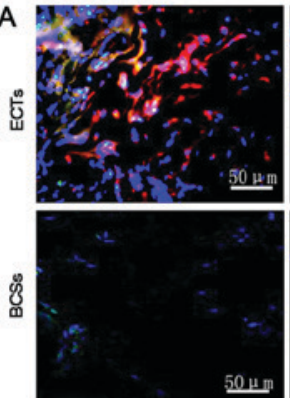

20 days

C
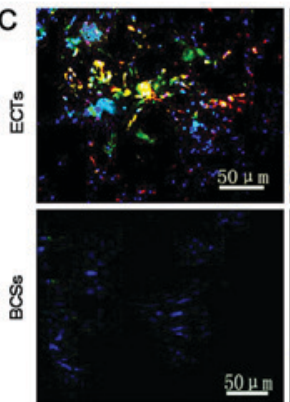

20 days
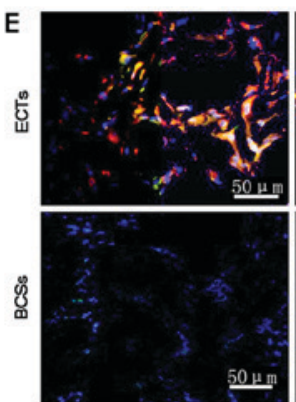

20 days
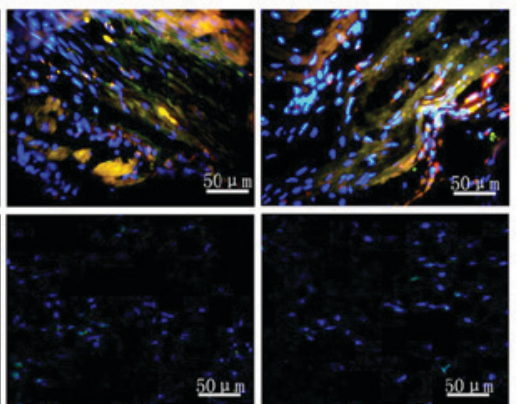

$\infty$ days
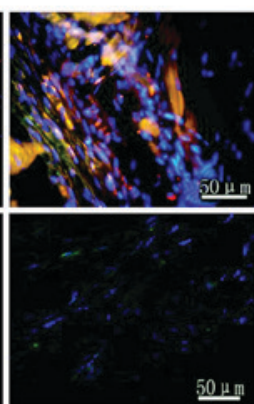

60 days
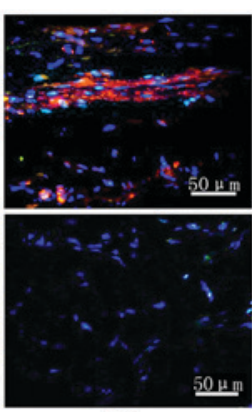

60 days
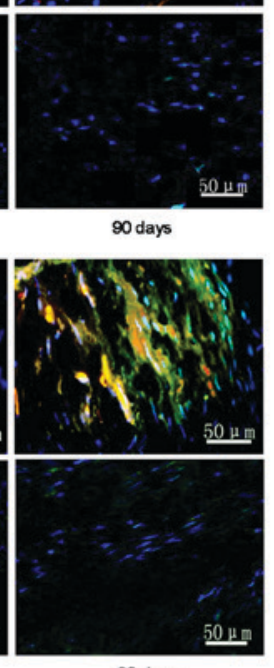

90 days
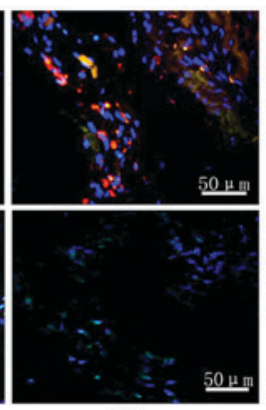

90 days
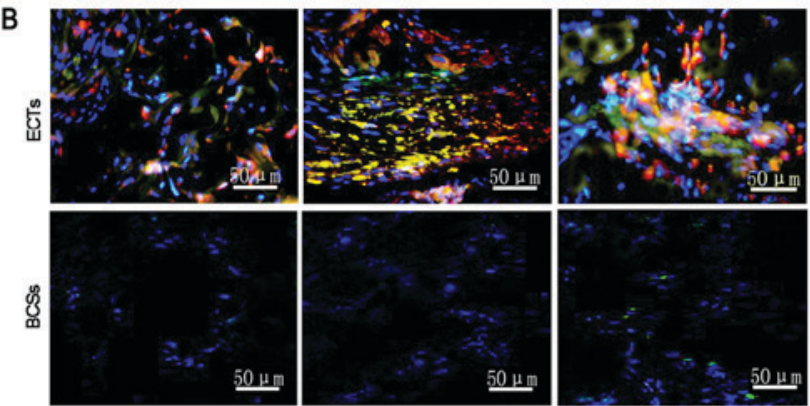

20 days
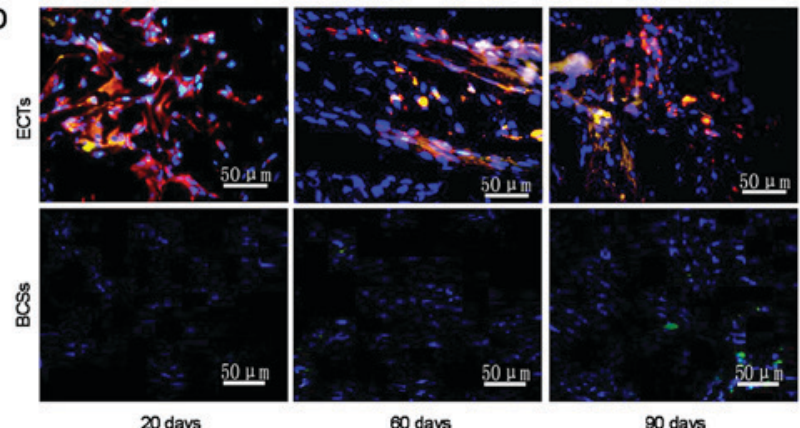

20 days
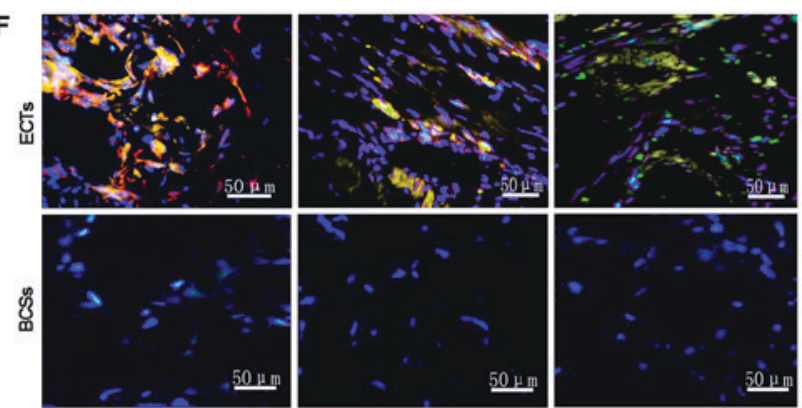

20 days

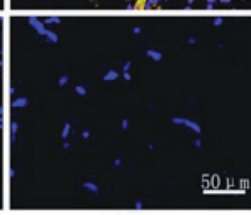

6 days

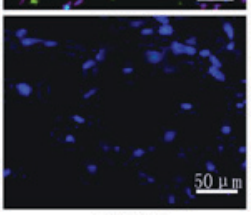

90 days

Figure 7. Immunostaining for connexin-40, connexin-43, HCN2, connexin-45, HCN4 and cTnT in the transplanted ECTs (n=26) and BCSs (n=24). Representative staining for (A) connexin-40, (B) connexin-43, (C) HCN2, (D) connexin-45, (E) HCN4 and (F) cTnT in the rats transplanted with ECTs and BCSs at days 20,60, and 90 after implantation. The transplanted tissue cells were identified by labeling with CM-Dil (red). Immunostaining for connexin-40, connexin-43, HCN2, connexin-45, HCN4 and cTnT in the implantation site was shown by the secondary antibodies (green). The nuclei were counterstained with DAPI (blue). ECTs, engineered conduction tissues; BCSs, blank collagen sponges.

period of 20 to 90 days post-transplantation. By contrast, the rats transplanted with BCSs did not exhibit any pre-excitation syndrome (Fig. 8).

To assess the functional conduction of ECTs in vivo, atrioventricular block was created by ethanol injection to induce atrioventricular node damage (Fig. 9). ECG recordings were used to monitor the rats with atrioventricular block. The results revealed that atrioventricular block was not recoverable within $1 \mathrm{~h}$ after the ethanol injection for all the normal rats(Table I). After $1 \mathrm{~h}$, increasing numbers of rats with atrioventricular block were restored. Consequently, it was confirmed that atrioventricular block in rats injected with ethanol could be maintainable or unrecoverable within $1 \mathrm{~h}$. However, the recovery rate in the rats implanted with ECTs was $61.54 \%$ within $1 \mathrm{~h}$ after atrioventricular block during the period of 20 to 90 days post-transplantation (Table II). The ECG recordings demonstrated that the cardiac rhythm of recovery was close to normal (Fig. 10). By contrast, the recovery rate was only $4.17 \%$ in the rats implanted with BCSs, and no rats in the Sham group exhibited atrioventricular block recovery (Table II). The recovery rate was significantly higher in the rats in the ECT group than in those in the BCS group or Sham group.

\section{Discussion}

Complete atrioventricular conduction block is a serious threat to human health. Despite the utility of electronic pacemakers in treating this disease, permanent implantation of devices is associated with certain complications. Recent studies have focused on the use of cell transplants and gene transfer for atrioventricular conduction block therapy $(10,11)$. However, neither gene transfer nor cellular transplantation reproduces the normal function of atrioventricular conduction. The aforementioned methods only provide palliation of atrioventricular conduction block. In particular, transgenic expression is transient and cells do not remain at the desired injection site. Therefore, the transplantation of ECTs may be ideal for the treatment of atrioventricular conduction block. The transplantation of ECTs into the heart may establish a novel atrioventricular conduction pathway, with characteristics similar to those of original conduction. Currently, little is 

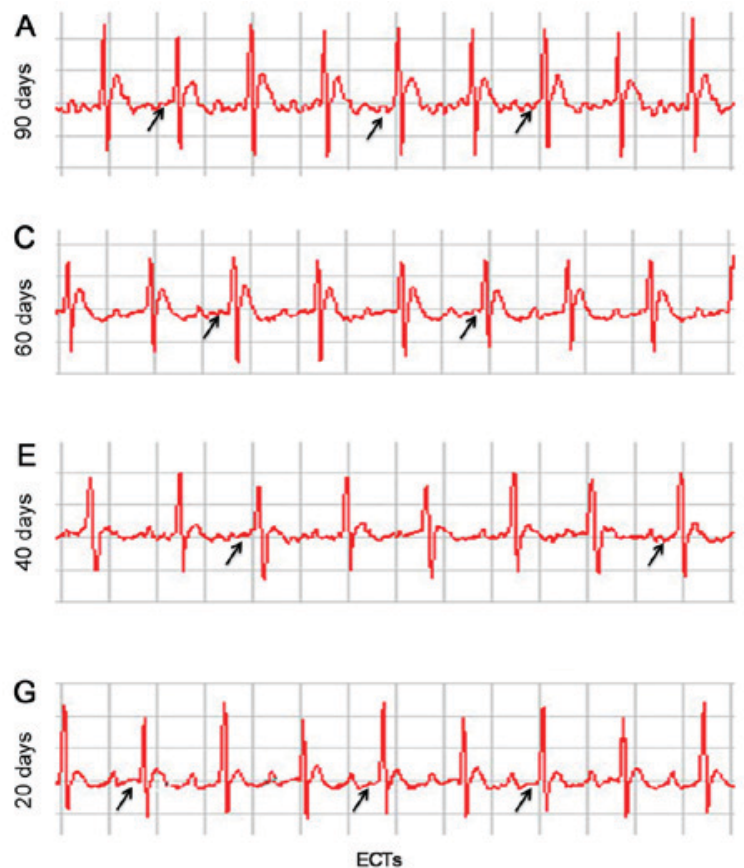

B

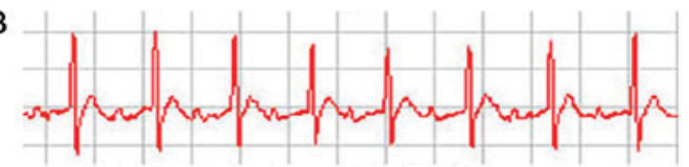

D

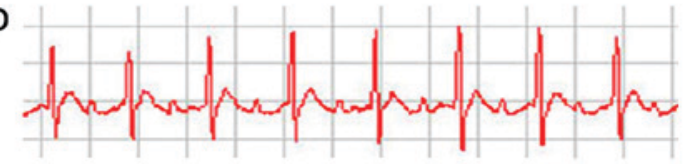

$\mathrm{F}$

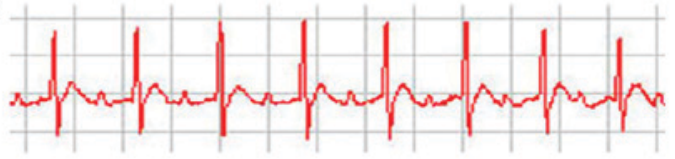

$\mathrm{H}$

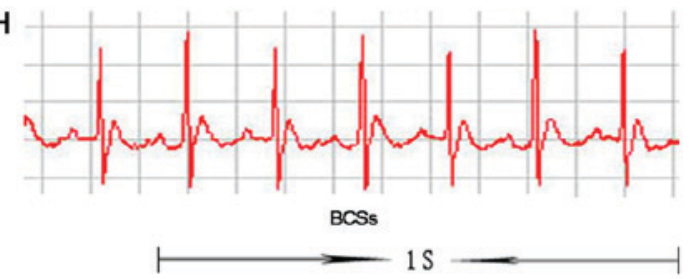

Figure 8. Pre-excitation syndrome is shown by ECG. Representative ECG (Lead II) obtained from the rats implanted with ECTs (n=26) at days (A) 20, (C) 40, (E) 60 and (G) 90 after implantation; and representative ECG (Lead II) obtained from the rats implanted with BCSs (n=24) at days (B) 20, (D) 40, (F) 60 and (H) 90 after implantation. The black arrows indicate the presence of the specific delta wave of the pre-excitation syndrome. ECG, electrocardiogram; ECTs, engineered conduction tissues; BCSs, blank collagen sponges.

A

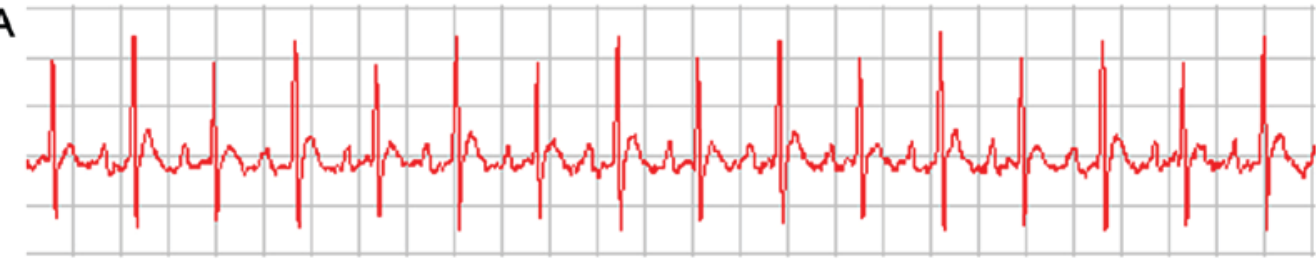

B

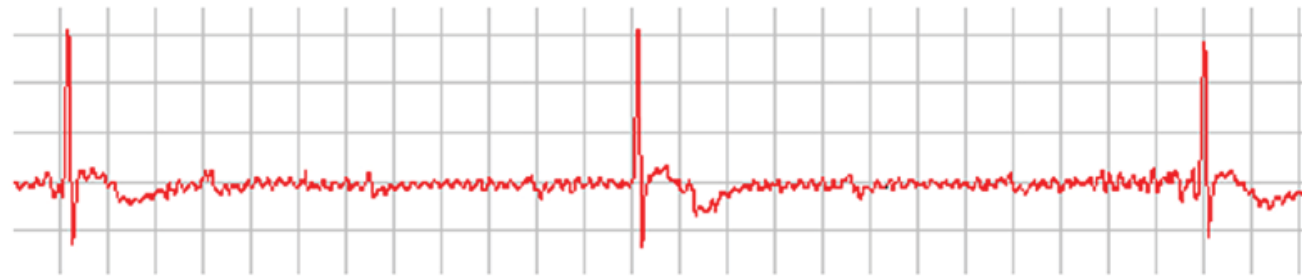

C

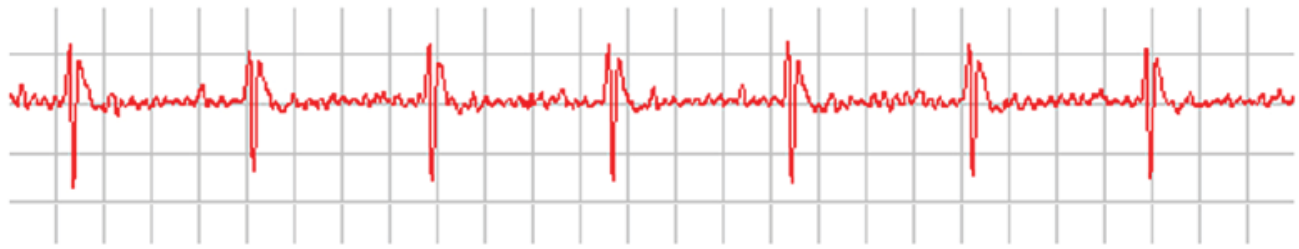

$1 \mathrm{~S}$

Figure 9. ECG (Lead II) obtained from the rats with atrioventricular block. (A) Representative ECG showing normal rhythm at a rate of approximately 400 bpm in the rats $(n=15)$ without atrioventricular block; $(B)$ representative ECG obtained from the rats $(n=7) 2 \mathrm{~h}$ after atrioventricular block; and $(\mathrm{C})$ representative ECG obtained from the rats $(n=6) 90$ days after atrioventricular block. ECG, electrocardiogram.

known regarding the transplantation and functional conduction of ECTs in vivo. In the present study, ECTs were created by seeding CPCs into collagen sponges in vitro. Next, ECTs were transplanted into the atrioventricular junction areas 
Table I. The time-dependent proportion of recovery in rats with atrioventricular block $(\mathrm{n}=85)$.

Time

\begin{tabular}{lcccccccccccc}
\cline { 2 - 8 } Proportion & $1 \mathrm{~h}$ & $2 \mathrm{~h}$ & $2 \mathrm{~d}$ & $4 \mathrm{~d}$ & $6 \mathrm{~d}$ & $8 \mathrm{~d}$ & $10 \mathrm{~d}$ & $14 \mathrm{~d}$ & $20 \mathrm{~d}$ & $40 \mathrm{~d}$ & $2 \mathrm{~m}$ & $3 \mathrm{~m}$ \\
\hline Recovery/block & $0 / 15$ & $1 / 7$ & $5 / 7$ & $3 / 7$ & $6 / 7$ & $4 / 6$ & $4 / 6$ & $4 / 6$ & $5 / 6$ & $4 / 6$ & $5 / 6$ & $5 / 6$ \\
\hline
\end{tabular}

Atrioventricular block was not recoverable only within $1 \mathrm{~h}$ following the ethanol injection for all the normal rats. After $1 \mathrm{~h}$, an increasing number of rats exhibited restored atrioventricular block. h, hour; d, day; $\mathrm{m}$, month.

A

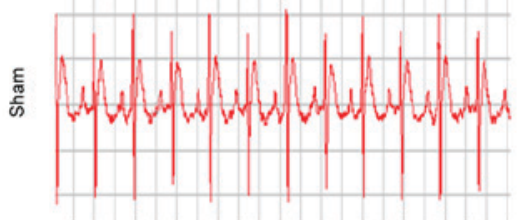

D

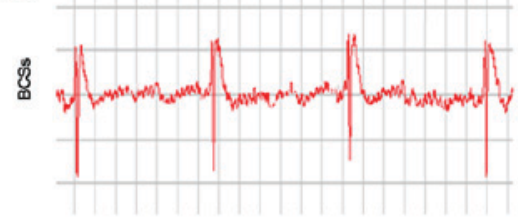

G

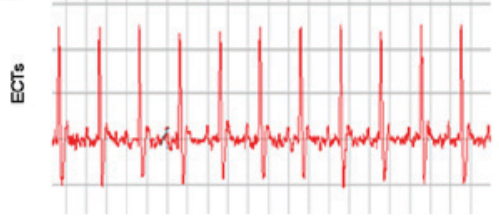

B

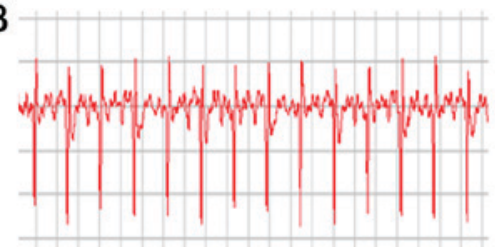

E

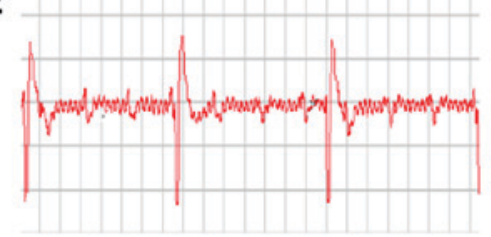

H

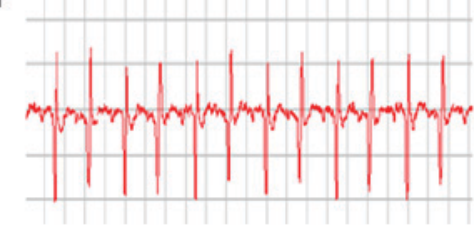

C

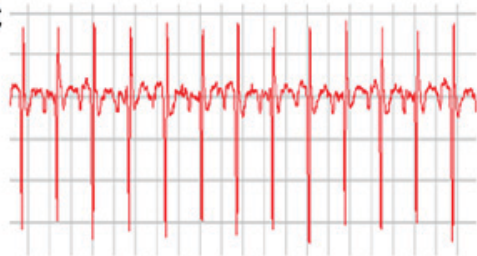

F

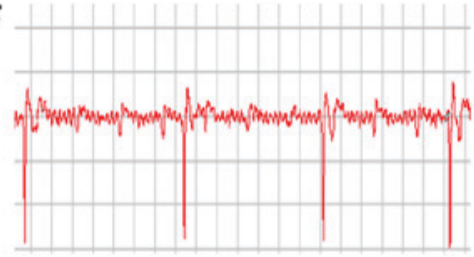

I

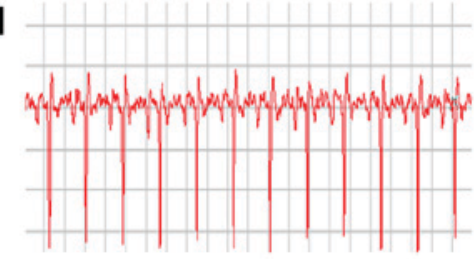

90 days

20 days

60 days

Figure 10. The recovery of atrioventricular block within one hour is shown in the rats implanted with ECTs. Representative ECG showing normal rhythm in the sham rats $(n=20)$ without atrioventricular block at days (A) 20, (B) 60 and (C) 90; (D-F) atrioventricular block within one hour is shown in the rats implanted with BCSs (n=24) at days (D) 20, (E) 60 and (F) 90; and the recovery of atrioventricular block within one hour was observed in the rats implanted with ECTs $(n=26)$ at days $(\mathrm{G}) 20,(\mathrm{H}) 60$ and (I) 90. ECG, electrocardiogram; ECTs, engineered conduction tissues; BCSs, blank collagen sponges.

Table II. Recovery rate in rats implanted with ETCs within $1 \mathrm{~h}$ following atrioventricular block.

\begin{tabular}{lcccc}
\hline Group & Recovery & Block & Total & Recovery rate, \% \\
\hline Sham $(n=20)$ & 0 & 20 & 20 & 0 \\
BCSs $(n=24)$ & 1 & 23 & 24 & 4.17 \\
ETCs $(n=26)$ & 16 & 10 & 26 & 61.54 \\
\hline
\end{tabular}

ECTs, engineered conduction tissues; BCSs, blank collagen sponges. The recovery rate was compared using the $\chi^{2}$ test, followed by Fisher's exact test. $\mathrm{P}<0.01$, values in the ETCs group vs. corresponding values in the BCSs group or the Sham group.

of rats in order to investigate the feasibility that they may restore atrioventricular block in a short amount of time.
In the present study, ECG recordings were used to detect atrioventricular conduction at days 20, 40,60 and 90 after transplantation. It was revealed that the specific delta wave of the pre-excitation syndrome appeared in the rats transplanted with ECTs at different time points during the transplantation period. The specific delta wave is mostly caused by the presence of the Kent bundle. Kent bundles are composed of atrial-like muscle and are mostly located between the left and right sides of the atrioventricular groove, connecting the atrial and ventricular myocytes $(12,13)$. The transplanted ECTs were able to establish an atrioventricular accessory pathway similar to Kent bundles between the atria and ventricles of rats.

Cardiac conduction pathway repair using engineered biological tissues has significant clinical translation potential. Previous studies have reported that myocardial tissue constructs may serve as conduits for restoring electrical conduction. The constructs were explanted into hearts for ex vivo experiments after 3 days $(14,15)$. Due to the short 
amount of time, it is difficult to validate in detail the structural and functional recoupling of the atrial and ventricular myocardia in these studies. The results of the present study provided evidence that the transplanted ECTs gradually developed an atrioventricular accessory pathway architecture during the period of 20 to 90 days post-transplantation. Histological staining confirmed the ECT survival and integration with the host cardiomyocytes in vivo. The cells and extracellular matrix were enriched in the transplanted ECTs, and the collagen sponges were increasingly degraded in this process. In particular, Masson's trichrome staining revealed that the transplanted ECTs gradually became homogeneous with the surrounding tissues. The staining is able to identify the fibrotic change of tissue. Collagen fibers were shown as blue and muscle fibers were shown as red. A large number of muscle fiber tissues were observed in the transplanted ECTs at 60 days post-transplantation. Poor conductive response has often been observed in fibrotic tissue. However, the number of fibers surrounding the ECTs was much less than that of native fibrotic tissue. Consequently, to the best of our knowledge, the fibrosis surrounding the ECTs did not influence the preexcitation pattern. These results provided essential histological evidence that the transplanted ECTs established an atrioventricular accessory pathway similar to Kent bundles.

Previously, we reported the characterization of CPCs derived from embryonic heart tubes (5-7). In these aforementioned studies, the characterization of CPCs was well investigated. The results revealed that $\mathrm{CPCs}$ are able to differentiate into cardiac cells, including cardiomyocytes, conduction cells and endothelial cells. Therefore, ECTs created by seeding CPCs into collagen sponges were able to develop into cardiac conduction or myocardial tissues in vivo in the present study. This deduction was consistent with the results obtained using immunofluorescence staining, which indicated that, following being transplanted into the rat hearts, the ECTs located between the atrial and ventricular myocytes exhibited positive staining for connexin-40, connexin-43, HCN2 and cTnT during the period of 20 to 90 days posttransplantation. By contrast, there was less positive staining for the aforementioned markers in the transplanted BCSs. As cardiac conducting tissue markers, connexin- 40 , connexin-43 and HCN2 were expressed in the transplanted ECTs. Positive staining for cTnT proved the existence of myocardial tissues in the transplanted ECTs. As cardiac nodal tissue markers, connexin-45 and HCN4 were rarely expressed in the transplanted ECTs, suggesting that the transplanted ECTs developed into cardiac conduction tissues with certain myocardial components. Connexins and pacemaker channel remodeling are usually considered a structural basis for the pattern of atrioventricular electrical conduction (16). The increased expression of connexin-40, connexin- 43 , HCN2 and $\mathrm{cTnT}$ provided adequate support for the atrioventricular accessory pathway in the present study. These proteins are also the indispensable molecular bases for atrioventricular electrical conduction $(17,18)$.

ECTs survived and mechanically integrated with the allogeneic myocardium following transplantation into rat hearts. The present study also provided evidence for the in vivo functional integration of ECTs by demonstrating the ability of ECTs to restore the normal rhythm of the heart in rats with atrioventricular block. ECTs established an atrioventricular accessory pathway by which $61.54 \%$ of the rats transplanted with ECTs had restored normal cardiac rhythm in $60 \mathrm{~min}$, while only $4.17 \%$ of the rats transplanted with BCSs had restored rhythm. As demonstrated in Fig. 10, the cardiac rhythm of recovery in the rats implanted with ECTs was close to normal. However, Fig. 8 revealed that pre-excitation patterns were observed intermittently, not in each beat. We hypothesized that this is because the preexcitation electrical signal was interfered with or interrupted by normal atrioventricular conduction without AV block (Fig. 8). Consequently, the patterns were observed transiently or intermittently prior to AV block. In summary, ECTs are a potential substitute therapy for atrioventricular conduction block.

\section{Acknowledgements}

The authors would like to thank Mrs. Sun Aijun and Mr. Qiao Liang (both of Department of Anatomy, Second Military Medical University) for providing valuable suggestions.

\section{Funding}

The present study was supported by grants from the National Natural Science Foundation of China (grant nos. 81371708 and 81571821) and by the Scientific Research and Innovation Project for College Students of Chongqing Medical University (2016-18).

\section{Availability of data and materials}

The datasets used and/or analyzed during the current study are available from the corresponding author on reasonable request.

\section{Authors' contributions}

$\mathrm{XZ}$ and SS conceived and designed the study and reviewed and edited the manuscript. WZ and XL performed the experiments, contributed to the data analyses and image processing, and wrote the manuscript. All the authors read and approved the final manuscript for publication.

\section{Ethics approval and consent to participate}

All the animal experiments were approved by the Ethics Committee of the Second Military Medical University (Shanghai, China).

\section{Patient consent for publication}

Not applicable.

\section{Competing interests}

The authors declare that they have no competing interests.

\section{References}

1. Cingolani E, Goldhaber JI and Marbán E: Next-generation pacemakers: From small devices to biological pacemakers. Nat Rev Cardiol 15: 139-150, 2018. 
2. Zhang X and Li XT: From theory to practice in biological pacing how to recreate an atrioventricular conduction pathway. Acad J Sec Mil Med Uni 38: 821-827, 2017.

3. Choi YH, Stamm C, Hammer PE, Kwaku KF, Marler JJ, Friehs I, Jones M, Rader CM, Roy N, Eddy MT, et al: Cardiac conduction through engineered tissue. Am J Pathol 169: 72-85, 2006.

4. Hou YB, Zou CW, Wang Y, Li DC, Li QB, Li HX, Zhang HZ, Zhang Q and Fan QX: Establishing a new electrical conduction pathway by anastomosis of the right auricle and right ventricle assisted by mesenchymal stem cells in a canine model. Transplant Proc 43: 3980-3986, 2011.

5. Zhang X, Zhang CS, Liu YC, Yang XQ, Xiong SH, Wen Y, Jiang EP, Li R, Zhang ZY, Liu F and Ye Y: Isolation, culture and characterization of cardiac progenitor cells derived from human embryonic heart tubes. Cells Tissues Organs 190: 194-208, 2009.

6. Zhang X, Guo JP, Chi YL, Liu YC, Zhang CS, Yang XQ, Lin HY, Jiang EP, Xiong SH, Zhang ZY and Liu BH: Endothelin-induced differentiation of $\mathrm{Nk} \times 2.5^{+}$cardiac progenitor cells into pacemaking cells. Mol Cell Biochem 366: 309-318, 2012.

7. Zhang X, Shen MR, Xu ZD, Hu Z, Chen C, Chi YL, Kong ZD, Li ZF, Li XT, Guo SL, et al: Cardiomyocyte differentiation induced in cardiac progenitor cells by cardiac fibroblast-conditioned medium. Exp Biol Med (Maywood) 239: 628-637, 2014.

8. Zhang L, Li X, Yu X, Li Y, Sun A, Huang C, Xu F, Guo J, Sun Y, Zhang X, et al: Construction of vascularized pacemaker tissues by seeding cardiac progenitor cells and endothelial progenitor cells into Matrigel. Life Sci 179: 139-146, 2017.

9. Lee RJ, Sievers RE, Gallinghouse GJ and Ursell PC: Development of a model of complete heart block in rats. J Appl Physiol (1985) 85: 758-763, 1998.

10. Cho HC: Pacing the heart with genes: Recent progress in biological pacing. Curr Cardiol Rep 17: 65, 2015.

11. Yokokawa M, Ohnishi S, Ishibashi-Ueda H, Obata H, Otani K, Miyahara Y,Tanaka K, Shimizu W, Nakazawa K, Kangawa K, et al: Transplantation of mesenchymal stem cells improves atrioventricular conduction in a rat model of complete atrioventricular block. Cell Transplant 17: 1145-1155, 2008.
12. Hanna Deschamps E and Hanna EB: Atrioventricular accessory pathways: Mechanisms, electrocardiograms, and associated arrhythmias. South Med J 109: 670-676, 2016.

13. Di Biase L, Gianni C, Bagliani G and Padeletti L: Arrhythmias involving the atrioventricular junction. Card Electrophysiol Clin 9: 435-452, 2017.

14. Cingolani E, Ionta V, Cheng K, Giacomello A, Cho HC and Marbán E: Engineered electrical conduction tract restores conduction in complete heart block: From in vitro to in vivo proof of concept. J Am Coll Cardiol 64: 2575-2585, 2014.

15. Kohl P: Structural and functional recoupling of atrial and ventricular myocardium: New conduits for electrical flow. J Am Coll Cardiol 64: 2586-2588, 2014

16. Nisbet AM, Camelliti P, Walker NL, Burton FL, Cobbe SM, Kohl P and Smith GL: Prolongation of atrio-ventricular node conduction in a rabbit model of ischaemic cardiomyopathy: Role of fibrosis and connexin remodelling. J Mol Cell Cardiol 94: 54-64, 2016

17. Yanni J, Maczewski M, Mackiewicz U, Siew S, Fedorenko O, Atkinson A, Price M, Beresewicz A, Anderson RH, Boyett MR and Dobrzynski H: Structural and functional alterations in the atrioventricular node and atrioventricular ring tissue in ischaemiainduced heart failure. Histol Histopathol 29: 891-902, 2014.

18. Bartos DC, Grandi E and Ripplinger CM: Ion channels in the heart. Compr Physiol 5: 1423-1464, 2015.

(c) (i) () $($ This work is licensed under a Creative Commons Attribution-NonCommercial-NoDerivatives 4.0 International (CC BY-NC-ND 4.0) License. 